\title{
Étude pilote des relations entre manifestations psychotiques et environnement de résidence
}

Antoine Longo, Hélène Charreire, Myriam Baron, Thierry Feuillet, Baptiste Pignon, Grégoire Baudin, Andrei Szoke et Franck Schurhoff

\section{CpenEdition}

Journals

Édition électronique

URL : https://journals.openedition.org/rfst/553

DOI : 10.4000/rfst.553

ISSN : 2492-3672

Traduction(s) :

Pilot study of the relationship between psychotic manifestations and living environment - URL

https://journals.openedition.org/rfst/757 [en]

Éditeur

Espaces et SOciétés (UMR 6590)

Référence électronique

Antoine Longo, Hélène Charreire, Myriam Baron, Thierry Feuillet, Baptiste Pignon, Grégoire Baudin, Andrei Szoke et Franck Schurhoff, «Étude pilote des relations entre manifestations psychotiques et environnement de résidence », Revue francophone sur la santé et les territoires [En ligne], Miscellanées, mis en ligne le 17 octobre 2020, consulté le 03 mai 2021. URL : http://journals.openedition.org/rfst/ 553 ; DOI : https://doi.org/10.4000/rfst.553

\section{cc) (†) (2)}

La Revue francophone sur la santé et les territoires est mise à disposition selon les termes de la Licence Creative Commons Attribution - Pas d'Utilisation Commerciale - Partage dans les Mêmes Conditions 4.0 International. 


\section{ETUDE PILOTE DES RELATIONS ENTRE MANIFESTATIONS PSYCHOTIQUES ET ENVIRONNEMENT DE RESIDENCE}

\section{LONGO Antoine}

Université Paris Est Créteil, LAB'URBA, F-94010 Créteil, France

CHARREIRE Hélène

Université Paris Est Créteil, LAB'URBA, F-94010 Créteil, France

\section{BARON Myriam}

Université Paris Est Créteil, LAB'URBA, F-94010 Créteil, France

\section{FEUILLET Thierry}

Université Paris 8, LADYSS, UMR 7533 CNRS, France

\section{PIGNON Baptiste}

AP-HP, Hôpital Henri Mondor, Département Medico-Universitaire de Psychiatrie et d'Addictologie (DMU IMPACT), Federation Hospitalo-Universitaire de Médecine de Precision (FHU ADAPT) F-94010, France ; INSERM U955, IMRB, Laboratoire Neuro-psychiatrie translationnelle, F-94010 Creteil, France; Fondation FondaMental, Créteil, 94000, France

\section{BAUDIN Grégoire}

AP-HP, Hôpital Henri Mondor, Département Medico-Universitaire de Psychiatrie et d'Addictologie (DMU IMPACT), Federation Hospitalo-Universitaire de Médecine de Precision (FHU ADAPT) F-94010, France ; INSERM U955, IMRB, Laboratoire Neuro-psychiatrie translationnelle, F-94010 Creteil, France; Fondation FondaMental, Créteil, 94000, France

\section{SZOKE Andrei}

AP-HP, Hôpital Henri Mondor, Département Medico-Universitaire de Psychiatrie et d'Addictologie (DMU IMPACT), Federation Hospitalo-Universitaire de Médecine de Precision (FHU ADAPT) F-94010, France ; INSERM U955, IMRB, Laboratoire Neuro-psychiatrie translationnelle, F-94010 Creteil, France; Fondation FondaMental, Créteil, 94000, France

\section{SCHURHOFF Franck}

AP-HP, Hôpital Henri Mondor, Département Medico-Universitaire de Psychiatrie et d'Addictologie (DMU IMPACT), Federation Hospitalo-Universitaire de Médecine de Precision (FHU ADAPT) F-94010, France ; INSERM U955, IMRB, Laboratoire Neuro-psychiatrie translationnelle, F-94010 Creteil, France ; Université Paris- Est Créteil (UPEC), Créteil, 94000, France ; Fondation FondaMental, Créteil, 94000, France

\footnotetext{
RESUME : Cet article aborde les déterminants des troubles psychotiques à travers l'étude des manifestations psychotiques "atténuées" (ou schizotypie quantitative); manifestations plus fréquentes que les troubles constitués (telle que la schizophrénie) mais rarement identifiés et pris en charge alors qu'ils impactent la qualité de vie. Si les déterminants individuels de ces troubles «atténués» sont plutôt bien connus, l'impact des déterminants contextuels (caractéristiques sociales, urbanistiques) des lieux de vie sur leur présence ou leur intensité n'a, par contre, pas encore été étudié. Les relations entre contexte de résidence et schizophrénie ont, en revanche, fait l'objet de recherches spécifiques.

A partir de données recueillies auprès de 255 adultes résidant dans trois quartiers de la commune de Créteil, cette étude s'intéresse aux relations entre les représentations (dont l'étendue) du quartier de résidence, les caractéristiques (socio-démographiques et urbanistiques) du quartier et les scores de schizotypie.
} 


\section{INTRODUCTION}

Les troubles mentaux représentent l'une des causes principales de morbidité et d'incapacité à l'échelle mondiale et se manifestent par de multiples formes telles que la dépression, les troubles bipolaires ou la schizophrénie (Organisation mondiale de la santé, 2013).

Selon I'OMS, la schizophrénie, une des pathologies mentales les plus sévères, affecterait plus de 23 millions de personnes dans le monde. En France, elle concerne 0,7\% de la population, soit environ 600.000 personnes (INSERM, 2014). La maladie s'accompagne le plus souvent d'un retentissement fonctionnel sévère sur le plan social, familial et professionnel. Par ailleurs, la prévalence des suicides chez les patients souffrant de schizophrénie est estimée entre 10 et $13 \%$ et celle des tentatives de suicide entre 20 et $50 \%$ (Besnier et al., 2009). Le risque de décès prématuré est augmenté de 40 à $60 \%$ chez les personnes souffrant de schizophrénie comparativement à la population générale (Organisation mondiale de la santé, 2013). Une récente étude française montre que le taux standardisé de décès prématuré (avant 65 ans) des personnes suivies pour un trouble psychiatrique est de 629,3 pour 100.000 habitants, contre 143,9 pour les bénéficiaires du régime général de l'Assurance maladie et des Sections Locales Mutualistes (SLM) ; soit un ratio de surmortalité prématurée de 4,4 (Coldefy \& Gandré, 2018). Les personnes suivies pour un trouble psychiatrique ont également une espérance de vie à 15 ans plus faible : de 48,9 ans pour les hommes (contre 65,3 ans pour les hommes non-suivis pour un trouble psychiatrique, soit 16,4 ans d'écart) et de 58,6 ans pour les femmes (contre 71,5 ans pour les femmes non-suivies pour un trouble psychiatrique soit un écart de 12,9 ans) (Caisse nationale d'assurance maladie (Cnam)). Ces différentiels ne seraient pas uniquement dus à une forte proportion de suicides, mais aussi à des maladies cardio-vasculaires, des cancers et/ou des infections qui seraient non seulement plus fréquents chez ces sujets, mais aussi plus tardivement dépistés et pris en charge.

Du fait du coût médico-économique des maladies mentales, une meilleure connaissance de leurs physiopathologies, des déterminants et I'amélioration de la prise en charge sont des enjeux majeurs de santé publique. Selon
I'Institut de Recherche et de Documentation en Economie de la Santé (IRDES), 10,6 \% du total des dépenses en santé hors-prévention ont été réalisés en France dans le secteur des troubles mentaux en 2006, soit le second domaine après les maladies de l'appareil circulatoire (12,6\%) (Fénina et al., 2006). En 2017, I'Assurance Maladie estime que les dépenses liées à la santé mentale représentent 23,2 milliards d'euros soit $14 \%$ des dépenses totales; ce qui en fait le second poste de dépenses derrière les épisodes hospitaliers (22\%), et le premier en termes de dépenses de santé (Caisse nationale d'assurance maladie (Cnam)). Par ailleurs, le coût indirect de la maladie, c'est-à-dire le coût lié aux dépenses sociales et médico-sociales, à l'accompagnement du malade, à la perte de productivité et à la baisse de la qualité de vie, est très important (Chevreul et al., 2013).

Au-delà des troubles les plus sévères comme la schizophrénie, l'étude des manifestations psychotiques dites «atténuées » (traits schizotypiques) représente également des enjeux cliniques et de santé publique (Ferchiou et al., 2017). Les manifestations psychotiques atténuées sont en effet beaucoup plus fréquentes que les troubles constitués (schizophrénie, trouble schizo-affectif) mais rarement identifiées et prises en charge alors qu'elles ont des conséquences sur la qualité de vie des sujets. Par ailleurs, «dans la mesure où les sujets présentant des scores élevés aux échelles de schizotypie sont à haut risque de développer un trouble du spectre schizophrénique » (Ferchiou et al., 2017), le dépistage de ces troubles en population générale permettrait de proposer des stratégies de prévention ciblées.

Pour toutes ces raisons, mieux comprendre les déterminants et les mécanismes sous-tendant ces manifestations psychotiques atténuées représente un objectif important de la recherche en santé mentale. L'hypothèse serait que ces manifestations psychotiques atténuées sont en partie influencées par des facteurs individuels et par des caractéristiques contextuelles liées à l'environnement de vie. Après l'étude historique de Faris et Dunham à Chicago démontrant qu'il n'y a pas d'homogénéité dans la distribution spatiale des troubles psychotiques (Faris \& Dunham, 1939), il a fallu attendre le début des années 2000 pour que les travaux de recherche sur les facteurs de risques individuels et contextuels des troubles 
mentaux s'intensifient (Kirkbride et al., 2014 ; Pignon et al., 2016 ; Weich et al., 2003). Cependant, si les facteurs individuels comme les facteurs de vulnérabilité génétique, les traumatismes psychologiques infantiles précoces, la consommation de cannabis, etc., sont aujourd'hui largement reconnus dans le développement des troubles psychotiques (Vilain et al., 2013), les facteurs contextuels, et plus particulièrement les caractéristiques de l'environnement urbain, restent peu explorés malgré l'intérêt qu'ils suscitent. C'est dans ce contexte que le projet «Géopsy » associant médecins, épidémiologistes et géographes a été élaboré. Son objectif général est de mieux connaître les déterminants urbains qui peuvent être associés à l'apparition de manifestations psychotiques atténuées (mesurées par une échelle de schizotypie quantitative) et plus spécifiquement de caractériser les variations géographiques du nombre de cas, ainsi que les caractéristiques environnementales associées à ces variations.

A partir de données recueillies au cours d'une enquête dans la commune de Créteil, nous nous sommes intéressés aux relations entre l'intensité des manifestations psychotiques atténuées (scores de schizotypie) et i) les représentations (dont l'étendue) du quartier de résidence et ii) les caractéristiques sociodémographiques et urbanistiques du quartier de résidence. Il s'agit ainsi de savoir si le fait de ne pas aimer vivre dans son quartier ou d'avoir une représentation restreinte de son quartier est associé à des manifestations psychotiques atténuées. Il s'agit également de déterminer à partir de données objectives - si les résidents de quartiers défavorisés sont plus susceptibles de développer des manifestations psychotiques atténuées que ceux des quartiers plus favorisés.

Cet article s'intéresse, en parallèle, à un enjeu méthodologique de délimitation et de caractérisation des quartiers perçus. L'objectif est de présenter la démarche développée, du recueil des limites des quartiers perçus aux relations identifiées en passant par les calculs des caractéristiques (sociales, urbanistiques) des quartiers.

\section{De la définition du quartier de résidence....}

Le mot « quartier » peut renvoyer à plusieurs définitions, car en géographie il n'a pas d'échelle ni de lieu propre (Humain-Lamoure, 2007). Les quartiers perçus, en référence aux études sur la région espace «vécu » d'Armand Frémont (Frémont, 1999), sont des espaces définis individuellement selon les limites de ce que I'on considère comme son « propre » quartier - qui dans notre cas est celui de résidence.

Une des étapes de l'étude était de recueillir les limites des quartiers de résidence perçues par les participants pour en caractériser l'environnement construit et social non plus à l'échelon d'une maille infra-communale (IRIS par exemple) ou d'une zone de dimension arbitraire (zone tampon autour du domicile) mais en tenant compte des représentations propres de l'espace de résidence de chaque sujet.

Au-delà de la composition sociale et de l'environnement construit (niveau socioéconomique, mixité d'usage des sols, densité de commerces), nous avons estimé la superficie de ces quartiers. Comme énoncé par Julie Vallée et ses co-auteurs (2010), un espace perçu limité « reflète le confinement spatial et social » et peut donc être associé à un risque plus élevé de manifestations psychotiques ou, à l'inverse, pourrait « être lié à un attachement profond au quartier de résidence et donc être associé à un risque plus faible » de manifestations psychotiques.

\section{.....à sa caractérisation}

Il n'existe pas, à notre connaissance, d'étude sur les relations entre manifestations psychotiques atténuées et caractéristiques du quartier de résidence. L'ensemble des recherches a porté sur les déterminants individuels et - dans une moindre mesure contextuels de la schizophrénie. La consommation de cannabis, le statut de migrant, la consommation de tabac et d'alcool ou encore une carence en vitamine $D$ sont les déterminants individuels identifiés comme étant les plus probants (Demily \& Thibaut, 2008).

Une revue de la littérature (Vilain et al., 2013) montre que l'urbanicité (urbanicity) est aujourd'hui identifiée comme un des facteurs de risque de la schizophrénie. Le terme d'urbanicité est utilisé pour qualifier les niveaux d'urbanisation - eux-mêmes qualifiés par différents indicateurs: «la définition opérationnelle de l'urbanicité est variable selon les études: nombre d'habitants dans la zone 
étudiée (petite ou grande ville), densité d'adresses, densité de population, etc. » (Vilain et al., 2013). De manière générale, l'incidence de la schizophrénie serait deux fois plus élevée dans les zones urbaines que dans les zones rurales (Kelly et al., 2010; Vilain et al., 2013, Szöke et al., 2014). Ainsi, dans l'étude de Van Os et al. (2004) réalisée au Danemark (cohorte nationale, 1020063 sujets), les sujets ayant une vulnérabilité familiale et qui vivent dans des zones urbaines ont une plus forte probabilité de développer des troubles que ceux qui vivent dans les zones plus rurales ${ }^{1}$ (Jim van Os et al., 2004). Si, selon Vilain et al. (2013), le niveau de preuve d'une association positive entre urbanisation et schizophrénie est solide, il est nécessaire de s'interroger sur la nature des éléments, au sein de ces environnements urbains, susceptibles d'influencer le développement de manifestations psychotiques.

\section{METHODOLOGIE}

\section{Terrain d'étude : trois « quartiers » de la commune de Créteil}

Notre terrain d'étude est la commune de Créteil, préfecture du département du Val-de-Marne, située au sud-est de Paris. Avec environ 91000 habitants (recensement INSEE, 2014) et une forte densité de population (7944 habitants $/ \mathrm{km}^{2}$ contre 5570 habitants $/ \mathrm{km}^{2}$ pour la moyenne départementale), Créteil est la commune la plus importante du département (et la $8^{\text {ème }}$ de la région Ile-de-France). Sa situation géographique et ses caractéristiques tant en termes de populations, d'habitats, d'équipements et d'urbanisme en font un site d'étude particulièrement pertinent pour tenter d'apporter des éléments de réponse à notre question de recherche.

A l'échelon infra-communal, Créteil est découpée en 30 Îlots Regroupés pour I'Information Statistique (IRIS) qui présentent d'importantes différences de tissu urbain et de niveau socio-économique. Trois IRIS ont été sélectionnés en raison de leurs profils socioéconomiques, démographiques et de leur localisation par rapport à la ligne de métro qui traverse la ville. Ces trois IRIS sont ceux de la Brèche, de la Habette et Casalis (Carte 1). Les IRIS de la Habette et Casalis sont, en grande partie, constitués de Quartiers en Politique de la Ville (QPV). L'IRIS de la Brèche est caractérisé par un niveau de revenu médian plus élevé (21 738 euros par unité de consommation) que ceux des IRIS de la Habette (13 920 euros) et Casalis (11 094 euros) ; le revenu médian étant de 18 742 euros à l'échelon communal.
${ }^{1}$ Le niveau d'urbanisation étant divisé en 5 catégories (capitale, banlieue proche de la capitale, ville de plus de 100000 habitants, ville entre 10000 et 100000 habitants, zone rurale). 


\section{Carte 1. Niveau de revenu médian des IRIS et principales caractéristiques (quartier politique de la ville, métro) à Créteil}

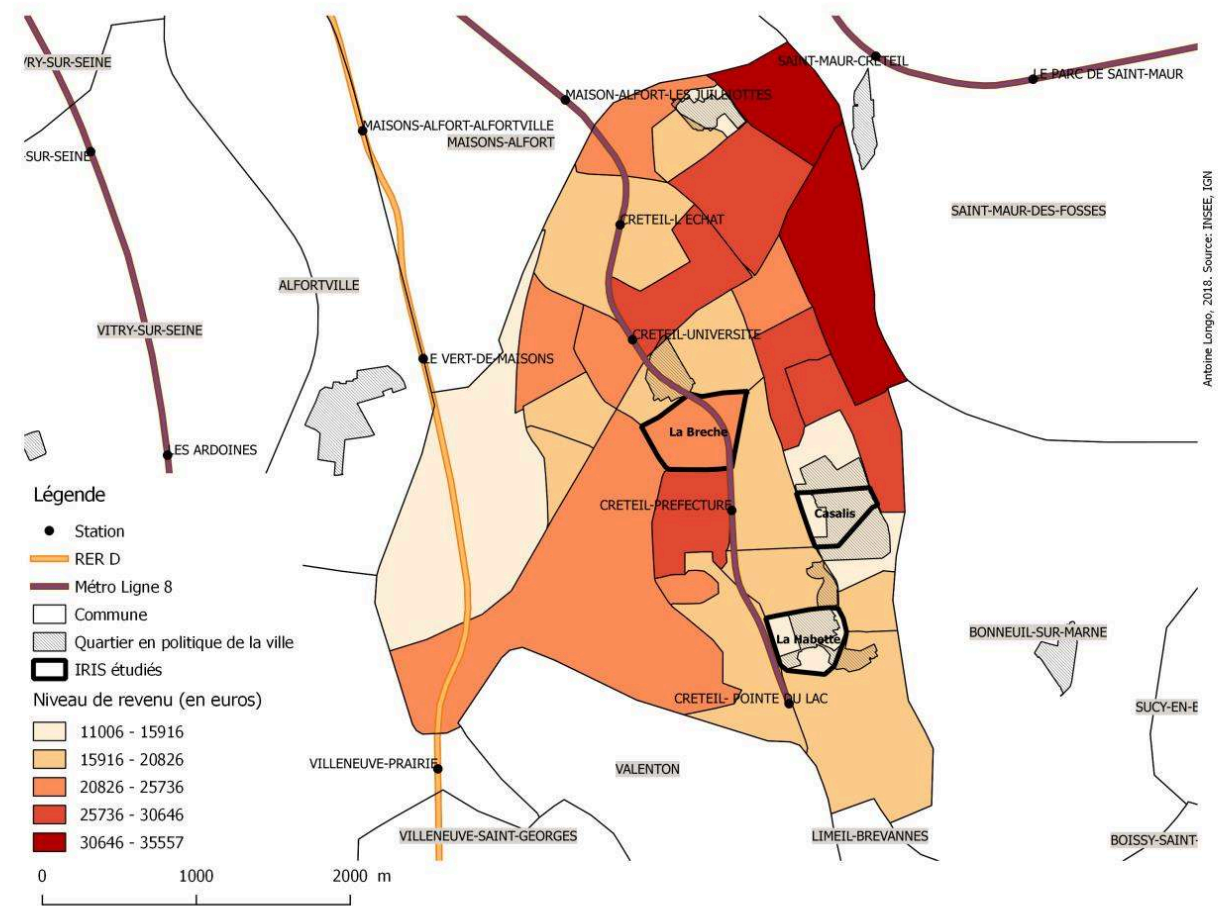

Les questionnaires ont été passés dans le cadre d'une enquête menée par la branche « santé » d'un institut de sondage sous la stricte supervision des investigateurs. La méthode des itinéraires a été utilisée pour la distribution des questionnaires. Cette méthode a consisté à parcourir, selon un plan établi au préalable à partir de cartes détaillées, l'ensemble des voies des IRIS de l'étude dans la commune de Créteil et de recenser l'ensemble des boîtes aux lettres. Celles-ci ont été listées de manière ordonnée selon leur emplacement sur l'itinéraire parcouru. Les adresses des ménages à enquêter ont ensuite été tirées au sort à partir du fichier contenant l'ensemble des adresses des ménages identifiées via les boîtes aux lettres. $\mathrm{Au}$ sein du ménage, un seul adulte a été échantillonné aléatoirement par l'enquêteur pour répondre au questionnaire. Lorsque la personne a refusé de participer à l'étude, l'ensemble du ménage a été exclu du recrutement. Les enquêtes à domicile ont été réalisées en semaine avant et après $17 \mathrm{~h}$ et en week-end (journée du samedi).

Les questionnaires ont permis de recueillir des informations sur l'état de santé, les relations sociales, les représentations et les pratiques selon les quartiers de résidence et sur les limites perçues du quartier de résidence auprès des résidents adultes des trois IRIS retenus durant I'année 2014. Le questionnaire de 8 pages était composé de six parties regroupant 106 questions. Les participants devaient renseigner leurs caractéristiques personnelles, incluant l'âge, le sexe, le lieu de naissance, le niveau de formation, la situation professionnelle et les revenus nets du foyer. La deuxième partie concernait le contexte de résidence, regroupant les questions relatives au logement de l'individu (nombre de pièces, prix du loyer), ainsi que les représentations du quartier de résidence (situation, réputation, bien-être au sein du quartier, etc). L'entourage de l'individu était évoqué dans la troisième partie pour évaluer l'isolement social. La santé perçue, le questionnaire de schizotypie quantitative et les comportements addictifs (consommation d'alcool, de tabac, de cannabis) étaient renseignés dans une quatrième partie. La cinquième partie questionnait les personnes enquêtées sur les antécédents familiaux concernant les problèmes de santé mentale (problèmes nerveux, émotionnels, psychologiques dans la famille). Enfin, la sixième et dernière partie était consacrée à la délimitation manuelle du quartier de résidence (sur une carte papier) tel que perçu par la personne interrogée. 
236 personnes ont répondu à ces questionnaires parmi lesquelles 9 ont retourné des cartes vierges de leur quartier de résidence et 5 ont indiqué des quartiers perçus de $10 \mathrm{~km}^{2}$ ou plus. L'échantillon final est donc de 225 sujets.

\section{Mesurer les manifestations psychotiques}

Les manifesfations psychotiques atténuées (schizotypie quantitative) sont évaluées à partir de la validation française d'un autoquestionnaire (Schizotypal Personality Questionnaire- Brief- Likert format (SPQ-B), 22 items) qui permet une approche quantitative (psychométrique) considérant l'ensemble des traits schizotypiques à travers trois dimensions distinctes pour tenir compte de la nature multifactorielle de la schizotypie ; c'est-à-dire la dimension positive ou cognitive-perceptive (comprenant des expériences perceptives inhabituelles et des croyances bizarres), la dimension négative ou sociale-interpersonnelle (caractérisée par la pauvreté des affects, l'absence d'amis proches et une anxiété sociale excessive) et la dimension qui relève de la désorganisation (comportement et discours bizarres) (Ferchiou et al., 2017). Les scores correspondant à ces 3 dimensions ainsi que la somme de l'ensemble des scores (score total) ont été utilisés dans notre recherche. Les scores obtenus aux échelles de schizotypie (score et sous-scores) reflètent la magnitude des manifestations psychotiques.

\section{Qualifier et délimiter les quartiers perçus}

Le contexte socio-économique des quartiers perçus a été caractérisé à partir du niveau médian de revenu issu du recensement INSEE (2012) au niveau des IRIS. Le contexte urbanistique des quartiers perçus a été caractérisé par les densités de commerces, d'espaces verts et la diversité d'occupation des sols (land use mixed index). La densité de commerces a été évaluée à partir de la base «équipement et service particuliers » de I'INSEE (2015), puis calculée en rapportant le nombre de commerces à la superficie du quartier (en $\mathrm{km}^{2}$ ). Les mesures de densité d'espaces verts et de diversité d'occupation des sols sont issues du mode d'occupation des sols (MOS) de I'Ile-de-France en 11 postes (Institut d'Aménagement et d'Urbanisme d'Île-deFrance, 2012). Dans notre étude, la part d'espaces verts sur le territoire a été estimée à partir du poste nommé « espaces ouverts et artificialisés » et l'indice de la diversité d'occupation des sols est le résultat du calcul suivant à partir des 11 postes :

Diversité d'occupation des sols $=-\sum_{i=1}^{11} p_{i} \ln p_{i}$ / $\ln (11)$

où $i$ est un type d'occupation des sols (allant ici de 1 à 11) et $p_{i}$ est la proportion de surface d'un de ces types par rapport à la surface totale de l'unité spatiale étudiée (Frank et al., 2006). L'indice est compris entre 0 et 1,0 signifiant que l'espace observé est utilisé à 100\% par le même poste et 1 signifiant que l'espace observé est équitablement occupé par les 11 postes.

Plusieurs étapes ont été nécessaires pour caractériser les quartiers de résidence tels que définis par chaque enquêté et non plus à partir des limites administratives des IRIS de résidence. Dans un premier temps, chaque dessin de quartier représenté sur une carte papier par l'enquêté a été numérisé dans Google-Earth puis intégré dans un Système d'Information Géographique (SIG).

Dans un second temps, les données du recensement de la population qui n'étaient disponibles qu'au niveau des IRIS ont été transformées en couche raster puis désagrégées à l'échelon de carreaux (nommés GRID). Le carroyage INSEE qui découpe géométriquement la France en carreaux de $1 \mathrm{~km}$ sur $1 \mathrm{~km}$, eux-mêmes divisés en 25 carreaux de $200 \mathrm{~m}$ sur $200 \mathrm{~m}$ a été utilisé pour cette étape (Figure 1). La densité d'espaces verts et l'indice de diversité d'occupation des sols ont aussi été rapportés à l'échelon des GRID. Dans un dernier temps, la moyenne des valeurs des carreaux qui compose chaque quartier perçu a été calculée pour chacune des variables du contexte de résidence. 
Figure 1. Limites des quartiers perçus dessinées par les 225 personnes interrogées dans les quartiers d'étude de la ville de Créteil (Val-de-Marne)

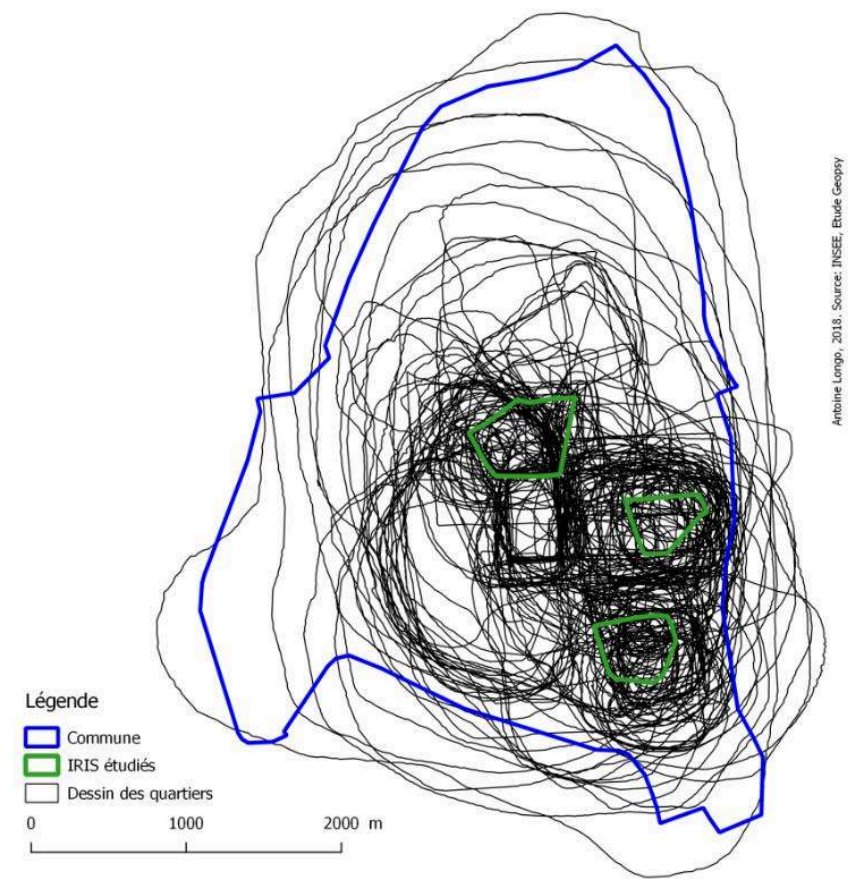

\section{Traitements statistiques}

Les superficies des quartiers perçus ont été décomposées en terciles; le premier tercile regroupe les superficies de quartier perçu les plus faibles (moins de $0,30 \mathrm{~km}^{2}$ ), le second tercile comprend les quartiers entre $0,30 \mathrm{~km}^{2}$ et $0,71 \mathrm{~km}^{2}$ tandis que le troisième tercile est constitué des quartiers de plus de $0,71 \mathrm{~km}^{2}$. Les caractéristiques objectives de l'environnement (densité de commerces, d'espaces verts, le niveau de revenu et l'indice de mixité urbaine) ont elles aussi été divisées en terciles. La variable « situation par rapport au quartier » est constituée de 3 modalités: (1) je souhaite continuer à vivre dans mon quartier, (2) je n'ai pas de souhait particulier et (3) je souhaite déménager. La variable « réputation du quartier » quant à elle est déclinée en deux modalités : (1) très bonne et bonne réputation et (2) mauvaise et très mauvaise réputation.

L'ensemble des analyses a été ajusté sur le sexe et l'âge des participants. Des analyses de variance (ANOVA) ont été utilisées afin de comparer les moyennes des scores de schizotypie en fonction de la superficie des quartiers perçus et en fonction des caractéristiques de l'environnement construit et perçu. Pour établir la significativité des résultats, le seuil de $5 \%$ a été retenu.

\section{RESULTATS}

\section{Profils des participants}

Dans notre échantillon de 225 personnes, on comptabilise légèrement plus de femmes que d'hommes (54,2 \% de femmes). L'âge moyen des enquêtés est de 52,7 ans (sachant que la personne interrogée la plus jeune a 19 ans et la plus âgée 91 ans). La majorité des enquêtés est originaire de France $(62,2 \%)$ et moins d'un tiers provient du continent africain (27,6 \%). 77 personnes ont été interrogées dans I'IRIS de La Brèche, 74 dans celui de Casalis et 74 dans celui de La Habette (Table 1). L'échantillon des personnes interrogées à La Brèche est le plus âgé avec une moyenne de 57,2 ans, tandis que celui des personnes interrogées à La Habette est le plus jeune avec une moyenne de 49 ans. 
Tableau 1. Caractéristiques socio-démographiques des 225 personnes enquêtées à Créteil

\begin{tabular}{|c|c|c|c|c|c|c|c|c|c|}
\hline & & \multicolumn{2}{|c|}{ Total } & \multicolumn{2}{|c|}{ La Breche } & \multicolumn{2}{|c|}{ Casalis } & \multicolumn{2}{|c|}{ La Habette } \\
\hline & & $\mathbf{N}$ & $\%$ & $\mathrm{~N}$ & $\%$ & $\mathbf{N}$ & $\%$ & $\mathrm{~N}$ & $\%$ \\
\hline \multicolumn{10}{|l|}{ Genre } \\
\hline & Femme & 128 & 56,9 & 42 & 54,6 & 44 & 59,5 & 42 & 56,8 \\
\hline & Homme & 97 & 43,1 & 35 & 45,5 & 30 & 40,5 & 32 & 43,2 \\
\hline $\begin{array}{l}\text { Age (moyenne } \\
\text { (écart type)) }\end{array}$ & & $\begin{array}{c}52,7 \\
(18,0)\end{array}$ & & $\begin{array}{c}57,2 \\
(19,3)\end{array}$ & & $\begin{array}{c}51,8 \\
(15,4)\end{array}$ & & $\begin{array}{c}49,0 \\
(18,3)\end{array}$ & \\
\hline \multicolumn{10}{|c|}{ Région de naissance } \\
\hline & France & 140 & 62,2 & 49 & 63,6 & 38 & 51,4 & 56 & 71,6 \\
\hline & Europe autre & 10 & 4,4 & 2 & 2,6 & 3 & 4,1 & 5 & 6,8 \\
\hline & Afrique du Nord & 36 & 16,0 & 14 & 18,2 & 13 & 17,6 & 9 & 12,2 \\
\hline & Afrique autre & 26 & 11,6 & 6 & 7,8 & 15 & 20,3 & 5 & 6,8 \\
\hline & Autre continent & 13 & 5,8 & 6 & 7,8 & 5 & 6,8 & 2 & 2,7 \\
\hline \multicolumn{10}{|c|}{ Type de logement } \\
\hline & Collectif & 224 & 99,6 & 76 & 100,0 & 74 & 100,0 & 74 & 100,0 \\
\hline & Données manquantes & 1 & 0,4 & & & & & & \\
\hline \multicolumn{10}{|l|}{ Statut marital } \\
\hline & En couple & 115 & 51,6 & 44 & 57,9 & 33 & 44,6 & 38 & 52,1 \\
\hline & Célibataire & 48 & 21,5 & 12 & 15,8 & 17 & 23,0 & 19 & 26,0 \\
\hline & Divorcé/séparé & 35 & 15,7 & 11 & 14,5 & 14 & 18,9 & 10 & 13,7 \\
\hline & Veuf & 25 & 11,2 & 9 & 11,8 & 10 & 13,5 & 6 & 8,2 \\
\hline & Données manquantes & 2 & 0,8 & & & & & & \\
\hline \multicolumn{10}{|l|}{ Diplôme } \\
\hline & Aucun & 44 & 20,5 & 5 & 6,9 & 24 & 33,8 & 15 & 20,8 \\
\hline & Inférieur au Bac & 68 & 31,6 & 21 & 29,2 & 24 & 33,8 & 23 & 31,9 \\
\hline & Bac (pro, générale) & 41 & 19,1 & 19 & 26,4 & 10 & 14,1 & 12 & 16,7 \\
\hline & Supérieur au Bac & 62 & 28,8 & 27 & 37,5 & 13 & 18,3 & 22 & 30,6 \\
\hline & Données manquantes & 10 & 4,4 & & & & & & \\
\hline \multicolumn{10}{|c|}{ Statut professionnel } \\
\hline & En activité & 88 & 39,3 & 29 & 37,7 & 28 & 38,4 & 31 & 41,9 \\
\hline & Chômage/arrêt & 25 & 11,2 & 5 & 6,5 & 14 & 19,2 & 6 & 8,1 \\
\hline & Etudiant/lycéen & 14 & 6,3 & 3 & 3,9 & 3 & 4,1 & 8 & 10,8 \\
\hline & Retraité & 79 & 35,3 & 36 & 46,8 & 18 & 24,7 & 25 & 33,8 \\
\hline & Au foyer & 18 & 8,0 & 4 & 5,2 & 10 & 13,7 & 4 & 5,4 \\
\hline & Données manquantes & 1 & 0,4 & & & & & & \\
\hline \multicolumn{10}{|c|}{ Niveau de revenu (en $€$ par mois) } \\
\hline & $<800-999$ & 29 & 16,3 & 5 & 7,5 & 18 & 30,0 & 6 & 11,8 \\
\hline & $1000-1999$ & 75 & 42,1 & 25 & 37,3 & 26 & 43,3 & 24 & 47,1 \\
\hline & $2000-2999$ & 44 & 24,7 & 18 & 26,9 & 13 & 21,7 & 13 & 25,5 \\
\hline & $3000+$ & 30 & 16,9 & 19 & 28,4 & 3 & 5,0 & 8 & 15,7 \\
\hline & Données manquantes & 47 & 20,8 & & & & & & \\
\hline
\end{tabular}

Au sein de cet échantillon, la totalité des habitants loge dans un habitat collectif (sauf une personne), ce qui correspond aux valeurs attendues par rapport au profil urbain des IRIS de l'étude (les trois IRIS étant tous composés de plus de $90 \%$ de logements collectifs). Le statut professionnel des personnes est particulier, puisque $11,1 \%$ se sont déclarés au chômage ou en arrêt de travail de longue durée, ce qui est faible comparé aux taux observés à partir du recensement de la population pour les IRIS étudiés (13,2 \% pour I'IRIS la Bréche, 20,7 $\%$ pour celui de la Habette et $23,9 \%$ pour Casalis). Le reste de l'échantillon se compose de retraités $(35,3 \%$, soit plus d'un tiers des sondés) et de 6,3\% d'étudiants. 
Enfin, on observe que 16,3\% des personnes interrogées ont déclaré des revenus inférieurs à 1000 euros par mois (dont 6,4\% inférieurs à 800 euros par mois), ce qui représente une partie non négligeable de la population. Cependant, un peu plus d'un cinquième de l'échantillon n'a pas répondu à cette question (20,8\% de données manquantes), ce qui limite l'interprétation de cette information.
Les moyennes des scores de schizotypie ont été comparées entre terciles de superficie des quartiers perçus pris deux à deux.

Tableau 2. Scores moyens de schizotypie par groupe (tercile) de superficie des quartiers perçus après ajustement sur l'âge et le sexe ( $n=225$ sujets, Analyse de variance)

\begin{tabular}{|c|c|c|c|c|c|c|c|c|c|c|c|c|}
\hline \multirow[b]{2}{*}{ Superficie (tercile) } & \multicolumn{3}{|c|}{ Score total } & \multicolumn{3}{|c|}{$\begin{array}{l}\text { Positif } \\
\text { (Cognitif-perceptif) }\end{array}$} & \multicolumn{3}{|c|}{ Désorganisation } & \multicolumn{3}{|c|}{$\begin{array}{l}\text { Négatif } \\
\text { (Social-interpersonnel) }\end{array}$} \\
\hline & Moy. & Et & $p^{*}$ & Moy. & $\mathrm{Et}$ & $p$ & Moy. & Et & $p$ & Moy. & Et & $p$ \\
\hline Faible & 44,9 & 13,8 & & 16,0 & 5,8 & & 10,6 & 4,6 & & 18,3 & 5,9 & \\
\hline Moyenne & 46,8 & 13,0 & & 16,1 & 5,4 & & 11,0 & 4,3 & & 19,8 & 5,7 & \\
\hline \multirow[t]{2}{*}{ Elevée } & 39,0 & 12,4 & & 13,2 & 4,9 & & 8,9 & 3,2 & & 16,9 & 6,0 & \\
\hline & & & 0.0009 & & & 0.0013 & & & 0.0049 & & & 0.01111 \\
\hline
\end{tabular}

*Comparaison de l'ensemble des groupes

Les différences de moyenne observées entre les groupes sont significatives pour l'ensemble des scores (Tableau 2). Les scores de schizotypie sont significativement plus faibles dans le groupe des enquêtés qui ont représenté les quartiers perçus les plus larges en comparaison des deux autres groupes, où les sujets ont une perception plus réduite de leur quartier de résidence (et des scores plus élevés). La significativité a aussi été testée par paires. Les résultats montrent que les différences entre les groupes 1 (superficie perçue faible) et 3 (superficie perçue élevée) et entre les groupes 2 (superficie moyenne) et 3 (superficie élevée) sont significatives mais pas entre les groupes 1 (faible) et 2 (moyenne).
Dans un second temps, des comparaisons de moyennes des scores ont été réalisées pour l'ensemble des variables objectives de l'environnement construit et social estimées au niveau du quartier perçu, c'est-à-dire la densité commerciale, le taux d'espaces verts, le niveau de revenu médian et l'indice de mixité urbaine (Tableau 3). L'ensemble des associations n'est pas significatif, à l'exception de la densité de commerces (dans le cas du score total et du sous-score cognitif/perceptif positif) et pour les espaces verts dans le cas du sous-score interpersonnel/négatif. Autrement dit, les scores de schizotypie de notre population d'étude ne sont pas - à quelques exceptions près - significativement associés aux caractéristiques de l'environnement construit et social de résidence. 
Tableau 3. Scores moyens de schizotypie en fonction des caractéristiques de l'environnement construit et perçu après ajustement sur l'âge et le sexe $(\mathbf{n}=225$ sujets, analyse de variance)

\begin{tabular}{|c|c|c|c|c|c|c|c|c|c|c|c|c|}
\hline & \multicolumn{3}{|c|}{ Score total } & \multicolumn{3}{|c|}{$\begin{array}{l}\text { Positif } \\
\text { (Cognitif-perceptif) }\end{array}$} & \multicolumn{3}{|c|}{ Désorganisation } & \multicolumn{3}{|c|}{$\begin{array}{l}\text { Négatif } \\
\text { (Social-interpersonnel) }\end{array}$} \\
\hline & Moy & Et & $p^{*}$ & Moy & Et & $p$ & Moy & Et & $p$ & Moy & Et & $p$ \\
\hline \multicolumn{13}{|c|}{ Environnement construit et social } \\
\hline \multicolumn{13}{|c|}{ Densité de commerces (tercile) } \\
\hline Faible & 47.1 & 13,2 & & 16,6 & 5,7 & & 10,9 & 4,5 & & 19,6 & 5,5 & \\
\hline Moyenne & 42,2 & 12,9 & & 14,8 & 5,2 & & 9,9 & 3,9 & & 17,8 & 5,7 & \\
\hline \multirow[t]{2}{*}{ Elevée } & 41,8 & 13,6 & & 13,9 & 5,4 & & 9,8 & 3,9 & & 17,5 & 6,4 & \\
\hline & & & 0,025 & & & 0,022 & & & 0.180 & & & 0.063 \\
\hline \multicolumn{13}{|c|}{ Espace vert (tereile) } \\
\hline Faible & 45,7 & 13,1 & & 15,1 & 5,7 & & 10,9 & 4,4 & & 19,6 & 5,6 & \\
\hline Moyen & 43,7 & 13,7 & & 15,5 & 5,6 & & 9,9 & 4,1 & & 18,4 & 6,3 & \\
\hline \multirow[t]{2}{*}{ Elevé } & 41,4 & 13,3 & & 14,6 & 5,3 & & 9,8 & 3,8 & & 17,0 & 5,7 & \\
\hline & & & 0.101 & & & 0.578 & & & 0.122 & & & 0,015 \\
\hline \multicolumn{13}{|c|}{ Niveau de revenu (tercile) } \\
\hline Faible & 45,6 & 14,6 & & 16,1 & 6,1 & & 10,7 & 4,8 & & 18,9 & 6,1 & \\
\hline Moyen & 43,8 & 12,5 & & 15,1 & 4,9 & & 10,1 & 3,9 & & 18,6 & 5,5 & \\
\hline \multirow[t]{2}{*}{ Elevé } & 41,3 & 12,9 & & 14,1 & 5,3 & & 9,8 & 3,6 & & 17,5 & 6,2 & \\
\hline & & & 0.211 & & & 0.136 & & & 0.516 & & & 0,359 \\
\hline \multicolumn{13}{|c|}{ Indice mixité urbaine (tercile) } \\
\hline Faible & 41,5 & 14,1 & & 14,5 & 5,6 & & 9,7 & 4,2 & & 17,3 & 6,5 & \\
\hline Moyen & 45,0 & 13,1 & & 15,2 & 5,7 & & 10,5 & 4,4 & & 19,3 & 5,5 & \\
\hline \multirow[t]{2}{*}{ Elevé } & 44,3 & 12,9 & & 15,6 & 5,3 & & 10,3 & 3,8 & & 18,3 & 5,7 & \\
\hline & & & 0,265 & & & 0.445 & & & 0.476 & & & 0,135 \\
\hline \multicolumn{13}{|c|}{ Environnement perçu } \\
\hline \multicolumn{13}{|c|}{ Situation/quartier } \\
\hline Rester & 42,4 & 12,0 & & 14,8 & 5,0 & & 9,7 & 3,5 & & 17.8 & 5,7 & \\
\hline Pas de souhait & 38,2 & 15,5 & & 12,8 & 6,1 & & 9,1 & 4,5 & & 16,3 & 6,6 & \\
\hline \multirow[t]{2}{*}{ Déménager } & 46,3 & 14,1 & & 15,9 & 5,8 & & 11,0 & 4,7 & & 19,4 & 6,0 & \\
\hline & & & 0,018 & & & 0,052 & & & 0,038 & & & 0.050 \\
\hline \multicolumn{13}{|c|}{ Réputation du quartier ${ }^{\text {t/ }}$} \\
\hline Bonne & 42,2 & 12,6 & & 14,4 & 5,1 & & 9,8 & 3,7 & & 18,0 & 6,0 & \\
\hline \multirow[t]{2}{*}{ Mauvaise } & 46,6 & 14,9 & & 16,5 & 6,2 & & 11,1 & 4,9 & & 19,1 & 5,8 & \\
\hline & & & 0,024 & & & 0,011 & & & 0,026 & & & 0,225 \\
\hline
\end{tabular}

Moy: moyenne, Et: erreur-type

"Comparaison de l'ensemble des groupes

** Données manquantes $\alpha$ réputation du quartier " pour un sujet $(n=224)$

Dans une troisième étape sont analysées les relations entre les scores de schizotypie et des variables de représentations de l'environnement, c'est-à-dire la perception de la réputation du quartier et le souhait de rester vivre dans le quartier ou, au contraire, de déménager (Tableau 3). Le score total de schizotypie est significativement plus élevé dans le groupe des sujets qui souhaitent quitter le quartier par rapport aux groupes de sujets qui souhaitent rester dans le quartier ou qui n'ont pas de souhait particulier. Les différences ne sont pas significatives entre ces deux derniers groupes. Les sujets qui déclarent que leur quartier de résidence a une bonne réputation ont des scores moyens de schizotypie significativement plus faibles que les sujets qui estiment que le quartier a une mauvaise réputation - sauf pour le sous-score interpersonnel/négatif où la relation est non significative.

\section{DISCUSSION}

Dans notre échantillon, les scores moyens de schizotypie sont globalement plus faibles dans les groupes qui déclarent des quartiers perçus larges et qui ont une représentation positive de leur quartier de résidence (réputation du quartier, souhait de rester y vivre). Ce résultat est à rapprocher d'une recherche récente (Olsen et al., 2017) qui a analysé les relations entre les représentations des quartiers de résidence et les scores d'anxiété et de dépression chez plus de 2000 résidents de 8 quartiers (local authority areas) de la ville de Glasgow et de la vallée de Clyde, dans l'ouest de l'Ecosse. Les représentations étaient qualifiées au travers de 14 items regroupés en 3 dimensions : (i) crimes et actes de vandalisme, (ii) environnement social incluant la réputation du quartier et (iii) environnement construit. Il apparaît que les individus dont les représentations du quartier 
sont négatives en termes d'environnement social/réputation et d'environnement construit ont aussi déclaré des scores de dépression et d'anxiété plus élevés que ceux qui ont des représentations positives. L'association est aussi significative entre le score d'anxiété et les représentations en lien avec les crimes et les actes de vandalisme.

Comme énoncé en introduction, il n'existe pas, à notre connaissance, d'étude explorant le lien entre scores de schizotypie et caractéristiques objectives de l'environnement social et construit. Cependant, les relations entre I'incidence de certains troubles (dépression, anxiété, schizophrénie) et des caractéristiques environnementales tels que le désavantage social, l'isolement social, la fragmentation sociale ou le niveau d'urbanisation des quartiers ont fait l'objet d'une plus grande attention. Par exemple, une association positive a été mise en évidence entre la prévalence des troubles psychotiques au sens large (schizophrénie, trouble schizophréniforme, trouble schizoaffectif et trouble délirant chronique) et le niveau de désavantage économique mesuré par le taux de chômage et de ménages non propriétaires d'une voiture (au niveau des IRIS) chez 358 adultes répondant à un diagnostic de trouble psychotique et recevant un traitement antipsychotique dans les communes de Créteil et de Maisons-Alfort (Pignon et al., 2016). Les relations avec le niveau de fragmentation sociale (pourcentage de résidents dans le quartier depuis moins de 2 ans, part de personnes vivant seules) et avec la part de personnes nées à l'étranger ou étrangères ne sont, par contre, pas significatives. D'autres études ont mis en évidence des taux d'incidence plus élevés de schizophrénie ou de troubles psychotiques dans les quartiers avec moins de soutien social (évalué par des indicateurs de cohésion sociale et de fragmentation sociale) et/ou avec une défaveur socio-économique élevée (Bhavsar et al., 2014; Kirkbride et al., 2014; J. van Os et al., 2000; Zammit et al., 2010). Cependant, selon Vilain et al. (2013), ces relations restent peu concluantes dans le cas de la schizophrénie, en raison notamment de la grande variabilité des facteurs contextuels étudiés. Par ailleurs, ces facteurs contextuels étudiés sont le plus souvent ceux associés au lieu de résidence de l'individu au moment de l'étude. Or, les mobilités résidentielles seraient plus importantes chez ceux qui souffrent de manifestations psychotiques que dans la population générale (DeVerteuil et al., 2007).

Les relations entre les caractéristiques urbanistiques objectives et les différents troubles mentaux sont également sujettes à discussion et peu étudiées. Une revue de la littérature publiée en 2016 identifie 11 articles issus de 10 études menées entre 2000 et 2012 et focalisées sur cette relation notamment en lien avec la dépression et l'anxiété (Gong et al., 2016). Cette revue suggère que des éléments de l'architecture, la densité d'espaces verts, la mixité urbaine, l'activité industrielle ou encore la densité du trafic auraient un impact sur des indicateurs de dépression et d'anxiété. La présence d'espaces verts est, par exemple, identifiée comme négativement associée aux troubles mentaux (Araya et al., 2007; Wu et al., 2017). Deux recherches, I'une conduite dans la ville de Santiago au Chili (Araya et al., 2007) et I'autre dans le comté Neath Port Talbot (sud du Pays de Galles) au Royaume-Uni (Thomas et al., 2007) ont quantifié l'environnement construit (Residential Environment Assessment Tool, REAT Score) et analysé les relations avec les troubles mentaux courants. Dans l'étude chilienne, l'environnement construit est défini par 25 items relevés via des audits de terrains tels que l'état des routes, des trottoirs, la présence d'espace verts, de mauvaises odeurs et la présence d'équipements (écoles, services de santé, de loisirs, etc.). Une typologie de 4 profils de quartier (sectors) est définie à partir de ces caractéristiques: (i) la qualité générale de la zone ; (ii) les installations, le bruit et la circulation dans la zone ; (iii) les espaces verts publics ; (iv) les sites vides. Les auteurs montrent une relation significative entre le score individuel de troubles mentaux et la qualité générale de la zone et les sites vides même après ajustement sur les caractéristiques individuelles (âge, sexe, statut marital, niveau d'éducation et niveau de revenu). Ainsi, les individus qui résident dans des quartiers dont l'environnement est de meilleure qualité ont un plus faible score de troubles mentaux. $A$ contrario, les personnes qui vivent dans des quartiers caractérisés par le profil (iv) sites vides ont un score de troubles mentaux plus important. Dans l'étude britannique, l'environnement construit des quartiers (postcode) est caractérisé par un score (divisé en terciles) basé sur 28 items relevés par audits de terrain telles que la présence de haies et de clôtures, d'espaces verts, etc. (Thomas et al., 
2007). Un score d'accessibilité aux équipements (de loisirs, sportifs, transports, services) est aussi calculé. Après ajustement sur les caractéristiques individuelles, les relations entre l'environnement et les troubles mentaux ne sont pas significatives.

Ainsi, il semblerait que - dans les rares études existantes - l'impact du contexte de résidence sur les troubles mentaux soit à rechercher davantage du côté des représentations des contextes de résidence et des caractéristiques sociales (individuelles et contextuelles) que des dimensions urbanistiques objectives (présence d'équipements, morphologie urbaine). Il serait toutefois nécessaire de rechercher davantage du côté de caractéristiques objectives du contexte de résidence qui n'ont pas été incluses dans notre étude, et plus particulièrement des expositions environnementales notamment sonores et des composantes sociales des quartiers. Une récente revue de la littérature souligne en effet le manque de données probantes sur les effets des nuisances sonores sur la santé mentale (Clark \& Paunovic, 2018).

Toutes les analyses des relations entre troubles mentaux et caractéristiques socioenvironnementales contextuelles sont réalisées au niveau du quartier de résidence défini par des limites administratives, que ce soit des secteurs (Araya et al., 2007) ou des LSOA (Wu et al., 2017) au Royaume-Uni, des census tracts aux Etats-Unis (Silver et al., 2002), ou des IRIS en France (Szoke et al., 2016). Ce constat mène à deux interrogations: la première sur la définition, ou plutôt la délimitation, du quartier de résidence et la seconde sur l'usage exclusif du quartier de résidence pour évaluer l'exposition à des déterminants contextuels. En effet, si la question de la définition du quartier n'est pas nouvelle en géographie ni en sociologie (Authier et al., 2007 ; Di Meo, 1994), identifier ses limites est toujours un enjeu dans la mesure où « le quartier resterait donc à lieu et à géométrie variables, constant défi au géographe » (Humain-Lamoure, 2007). Des travaux récents montrent le décalage entre les quartiers définis par les individus (self-defined ou perceived neighbourhood dans la littérature internationale) et les quartiers pré-définis par les limites administratives ou par des zones tampons (Charreire et al., 2016 ; Vallée et al., 2015). Or, la très grande majorité des recherches s'appuie sur ces limites prédéfinies pour évaluer l'exposition à des déterminants contextuels de santé, créant ainsi d'éventuels biais d'exposition: "the practice of adopting administrative definitions of neighborhoods (census tracts in US research, electoral wards in UK research) inevitably results in exposure misclassification unless the boundaries of relevant neighborhood dimensions (e.g., service delivery, social networks) happen to coincide exactly with them. To the extent that the misclassification is non differencial with respect to the outcomes, true neighborhood effects will be underestimated' (Kawachi \& Berkman, 2004).

L'impact de ces divergences a été estimé dans des études évaluant la présence de ressources en santé (Vallée et al., 2014), de marchés alimentaires (Crawford et al., 2014) et de caractéristiques urbaines comme les aménités en lien avec la marche (Smith et al., 2010; Stewart et al., 2015). D'autres recherches montrent la variabilité des indicateurs de marchabilité tels que la densité résidentielle, I'usage des sols et la connectivité en fonction des différentes limites des quartiers (quartier perçu, zone tampon euclidienne et réseau) (Bödeker, 2018; Stewart et al., 2015). Cependant, à notre connaissance, aucune analyse n'a été réalisée sur les relations entre quartiers perçus et indicateurs de santé. Par ailleurs, comme rappelé par Graif et al. (2016), les études sur les effets de quartier sur la santé traitent souvent les quartiers comme s'ils étaient des îles isolées (Graif et al., 2016). Or, pour comprendre ces effets, il est nécessaire de tenir compte des espaces situés autour du quartier de résidence (Graif et al., 2016) et plus largement des espaces d'activités (Vallée et al., 2011).

\section{CONCLUSION}

Dans notre échantillon d'étude, des relations apparaissent entre les représentations du quartier de résidence, notamment sa taille, et les scores de schizotypie. Néanmoins, et contrairement à nos hypothèses, la plupart des variables objectives du contexte de résidence qui ont été retenues et analysées dans cette recherche ne sont pas associées aux scores de schizotypie. Ce résultat peut s'expliquer, en partie, par (i) la faible variabilité des scores de schizotypie et des caractéristiques urbanistiques entre les trois quartiers de la ville de Créteil et (ii) l'absence de caractéristiques telle que l'exposition à des nuisances environnementales 
(notamment sonores) ou à des délits et actes de vandalisme par exemple. Cependant, l'analyse transversale - réalisée auprès d'un échantillon de 225 adultes non-représentatif de la population générale - ne permet pas de généraliser ces résultats ni d'établir des relations de causalité. Il n'a ainsi pas été possible d'évaluer les interrelations entre les troubles psychiques et les espaces vécus et perçus ou, en d'autres termes, comment les troubles psychiques peuvent, eux aussi, modifier les pratiques et/ou les représentations des environnements de vie.

Suite à cette étude pilote, deux pistes de recherche sont proposées. La première serait de renouveler les analyses entre différentes villes/quartiers avec des morphologies urbaines et des compositions sociales contrastées idéalement sur des temps longs pour prendre en compte l'évolution des contextes urbains tout comme le parcours de vie des individus en incluant les expositions aux nuisances sonores. En effet, la dimension temporelle est à prendre en compte, que ce soit pour le quartier dont la réputation, les équipements, la structure sociale peuvent changer au cours du temps, ou pour les individus dont les expositions varient tout au long de la vie en fonction des lieux de résidence (Mair et al., 2015 ; Olsen et al., 2017).

Une seconde piste serait d'évaluer plus précisément les représentations et les pratiques des populations en tant qu'éléments révélateurs des «vrais » usages des aménités et des espaces publics. Ces recherches permettraient de mieux connaître les déterminants participant à l'apparition des troubles psychotiques et de proposer des actions de prévention adaptées au contexte géographique.

\section{REMERCIEMENT}

Cette recherche a bénéficié d'un financement de I'Université Paris Est, Créteil (UPEC) dans le cadre d'appel à projet pluridisciplinaire. Nous remercions tout spécialement Virginie Mobillion, géographe, pour la coordination de l'étude de terrain et l'ensemble des personnes qui ont accepté de participer à cette étude.

\section{BIBLIOGRAPHIE}

Araya, R., Montgomery, A., Rojas, G., Fritsch, R., Solis, J., Signorelli, A., \& Lewis, G. (2007). Common mental disorders and the built environment in Santiago, Chile. British Journal of Psychiatry, 190(05), 394-401. https://doi.org/10.1192/bjp.bp.106.024596

Authier, J.-Y., Bacqué, M.-H., \& Guérin-Pace, F. (Eds.). (2007). Le quartier: Enjeux scientifiques, actions politiques et pratiques sociales. Paris: Éditions de la Découverte.

Besnier, N., Gavaudan, G., Navez, A., Adida, M., Jollant, F., Courtet, P., \& Lançon, C. (2009). Approche clinique du suicide au cours de la schizophrénie (I). Identification des facteurs de risque. L'Encéphale, 35(2), 176-181. https://doi.org/10.1016/j.encep.2008.02.009

Bhavsar, V., Boydell, J., Murray, R., \& Power, P. (2014). Identifying aspects of neighbourhood deprivation associated with increased incidence of schizophrenia. Schizophrenia Research, 156(1), 115-121. https://doi.org/10.1016/j.schres.2014.03.014

Bödeker, M. (2018). Walking and Walkability in Pre-Set and Self-Defined Neighborhoods: A Mental Mapping Study in Older Adults. International Journal of Environmental Research and Public Health, 15(7), 1363. https://doi.org/10.3390/ijerph15071363

Caisse nationale d'assurance maladie (Cnam). Améliorer la qualité du système de santé et maîtriser les dépenses. Propositions de I'Assurance Maladie pour 2020. (ameli.fr, rapports charges et produits pour 2020).

Charreire, H., Feuillet, T., Roda, C., Mackenbach, J. D., Compernolle, S., Glonti, K., Oppert, J.-M. (2016). Self-defined residential neighbourhoods: Size variations and correlates across five European urban regions: Selfdefined residential neighbourhoods. Obesity Reviews, 17, 9-18. https://doi.org/10.1111/obr.12380

Chevreul, K., Prigent, A., Bourmaud, A., Leboyer, M., \& Durand-Zaleski, I. (2013). The cost of mental disorders in France. European Neuropsychopharmacology, 23(8), 879-886. https://doi.org/10.1016/j.euroneuro.2012.08.0 12

Clark, C., \& Paunovic, K. (2018). WHO Environmental Noise Guidelines for the European Region: A Systematic Review on Environmental Noise and Quality of Life, Wellbeing and Mental Health. International Journal of Environmental Research and Public 
Health,

15(11),

2400.

https://doi.org/10.3390/ijerph15112400

Coldefy, M., \& Gandré, C. (2018). Personnes suivies pour des troubles psychiques sévères: Une espérance de vie fortement réduite et une mortalité prématurée quadruplée, [Questions d'économie de la santé $\left.n^{\circ} 237\right]$.

Crawford, T. W., Jilcott Pitts, S. B., McGuirt, J. T., Keyserling, T. C., \& Ammerman, A. S. (2014). Conceptualizing and comparing neighborhood and activity space measures for food environment research. Health \& Place, 30, 215-225.

https://doi.org/10.1016/j.healthplace.2014.09. 007

Demily, C., \& Thibaut, F. (2008). Facteurs de risque environnementaux à la schizophrénie. Annales Médico-psychologiques, revue psychiatrique, 166(8), 606-611. https://doi.org/10.1016/j.amp.2006.09.016

DeVerteuil, G., Hinds, A., Lix, L., Walker, J., Robinson, R., \& Roos, L. L. (2007). Mental health and the city: Intra-urban mobility among individuals with schizophrenia. Health \& Place, 13(2), 310-323. https://doi.org/10.1016/j.healthplace.2006.02. 001

Di Meo, G. (1994). Epistémologie des approches géographiques et socio-anthropologiques du quartier urbain. Annales de Géographie, 103(577), 255-275. https://doi.org/10.3406/geo.1994.13781

Faris, R., \& Dunham, H. (1939). Mental Disorders in Urban Areas: An Ecological Study of Schizophrenia and Other Psychoses (University of Chicago Press, Chicago).

Fénina, A., Geffroy, Y., Minc, C., Renaud, T., Sarlon, E., \& Sermet, C. (2006). Les dépenses de prévention et les dépenses de soins par pathologie en France. Bulletin d'information En Économie de La Santé, IRDES, (111).

Ferchiou, A., Todorov, L., Lajnef, M., Baudin, G., Pignon, B., Richard, J.-R., ... Schürhoff, F. (2017). Analyse de la structure factorielle de la version brève du questionnaire de personnalité schizotypique (SPQ-B) - format Likert - en population générale en France. L'Encéphale, 43(6), 558-563. https://doi.org/10.1016/j.encep.2016.05.011
Frank, L. D., Sallis, J. F., Conway, T. L., Chapman, J. E., Saelens, B. E., \& Bachman, W. (2006). Many Pathways from Land Use to Health: Associations between Neighborhood Walkability and Active Transportation, Body Mass Index, and Air Quality. Journal of the American Planning Association, 72(1), 75-87. https://doi.org/10.1080/01944360608976725

Frémont, A. (1999). La région, espace vécu. Paris: Flammarion.

Gong, Y., Palmer, S., Gallacher, J., Marsden, T., \& Fone, D. (2016). A systematic review of the relationship between objective measurements of the urban environment and psychological distress. Environment International, 96, 48-57. https://doi.org/10.1016/j.envint.2016.08.019

Graif, C., Arcaya, M. C., \& Diez Roux, A. V. (2016). Moving to opportunity and mental health: Exploring the spatial context of neighborhood effects. Social Science \& Medicine, 162, 50-58. https://doi.org/10.1016/j.socscimed.2016.05.0 36

Humain-Lamoure, A.-L. (2007). Le quartier comme objet en géographie. In Recherches. Le quartier: Enjeux scientifiques, actions politiques et pratiques sociales (pp. 41-51). Paris: Edition La Découverte.

INSERM. (2014). Schizophrénie: Intervenir au plus tôt pour limiter la sévérité des troubles. Retrieved from Dossier d'Information INSERM https://www.inserm.fr/information-ensante/dossiers-information/schizophrenie

Kawachi, I., \& Berkman, L. (2004). Neighbourhoods and Health. I and Berkman LF (eds). New York: Oxford University Press Inc, USA, 2003, pp. 320, 39.50. ISBN 0195138384. International Journal of Epidemiology, 34(1), 231-232. https://doi.org/10.1093/ije/dyh387

Kelly, B. D., O'Callaghan, E., Waddington, J. L., Feeney, L., Browne, S., Scully, P. J., ... Larkin, C. (2010). Schizophrenia and the city: A review of literature and prospective study of psychosis and urbanicity in Ireland. Schizophrenia Research, 116(1), 75-89. https://doi.org/10.1016/j.schres.2009.10.015

Kirkbride, J. B., Jones, P. B., Ullrich, S., \& Coid, J. W. (2014). Social Deprivation, Inequality, and the Neighborhood-Level Incidence of Psychotic 
Syndromes in East London. Schizophrenia Bulletin, 40(1), 169-180. https://doi.org/10.1093/schbul/sbs151

Mair, C., Diez Roux, A. V., Golden, S. H., Rapp, S., Seeman, T., \& Shea, S. (2015). Change in neighborhood environments and depressive symptoms in New York City: The Multi-Ethnic Study of Atherosclerosis. Health \& Place, 32, 93-98.

https://doi.org/10.1016/j.healthplace.2015.01. 003

Olsen, J., Dundas, R., \& Ellaway, A. (2017). Are Changes in Neighbourhood Perceptions Associated with Changes in Self-Rated Mental Health in Adults? A 13-Year Repeat CrossSectional Study, UK. International Journal of Environmental Research and Public Health, 14(12), 1473.

https://doi.org/10.3390/ijerph14121473

Organisation mondiale de la santé. (2013). Plan d'action global pour la santé mentale 20132020. OMS.

Pignon, B., Schürhoff, F., Baudin, G., Ferchiou, A., Richard, J.-R., Saba, G., ... Szöke, A. (2016). Spatial distribution of psychotic disorders in an urban area of France: An ecological study. Scientific Reports, 6(1). https://doi.org/10.1038/srep26190

Silver, E., Mulvey, E. P., \& Swanson, J. W. (2002). Neighborhood structural characteristics and mental disorder: Faris and Dunham revisited. Social Science \& Medicine (1982), 55(8), 1457-1470.

Smith, G., Gidlow, C., Davey, R., \& Foster, C. (2010). What is my walking neighbourhood? A pilot study of English adults' definitions of their local walking neighbourhoods. The International Journal of Behavioral Nutrition and Physical Activity, 7, 34. https://doi.org/10.1186/1479-5868-7-34

Stewart, T., Duncan, S., Chaix, B., Kestens, Y., Schipperijn, J., \& Schofield, G. (2015). A novel assessment of adolescent mobility: A pilot study. The International Journal of Behavioral Nutrition and Physical Activity, 12, 18. https://doi.org/10.1186/s12966-015-0176-6

Szoke, A., Pignon, B., Baudin, G., Tortelli, A., Richard, J.-R., Leboyer, M., \& Schürhoff, F. (2016). Small area-level variation in the incidence of psychotic disorders in an urban area in France: An ecological study. Social Psychiatry and Psychiatric Epidemiology, 51(7), 951-960. https://doi.org/10.1007/s00127-016$1231-6$

Thomas, H., Weaver, N., Patterson, J., Jones, P., Bell, T., Playle, R., ... Araya, R. (2007). Mental health and quality of residential environment. British Journal of Psychiatry, 191(06), 500-505. https://doi.org/10.1192/bjp.bp.107.039438

Vallée, J., Cadot, E., Roustit, C., Parizot, I., \& Chauvin, P. (2011). The role of daily mobility in mental health inequalities: The interactive influence of activity space and neighbourhood of residence on depression. Social Science \& Medicine, 73(8), 1133-1144. https://doi.org/10.1016/j.socscimed.2011.08.0 09

Vallée, J., Frohlich, K., Kestens, Y., \& Shareck, M. (2014). Accès aux ressources et santé mentale à Montréal. L'importance de la mobilité quotidienne. Métropolitiques. Retrieved from https://www.metropolitiques.eu/Acces-auxressources-et-sante.html

Vallée, J., Le Roux, G., Chaix, B., Kestens, Y., \& Chauvin, P. (2015). The 'constant size neighbourhood trap' in accessibility and health studies. Urban Studies, 52(2), 338-357. https://doi.org/10.1177/0042098014528393

van Os, J., Driessen, G., Gunther, N., \& Delespaul, P. (2000). Neighbourhood variation in incidence of schizophrenia. Evidence for person-environment interaction. The British Journal of Psychiatry: The Journal of Mental Science, 176, 243-248.

van Os, Jim, Pedersen, C. B., \& Mortensen, P. B. (2004). Confirmation of Synergy Between Urbanicity and Familial Liability in the Causation of Psychosis. American Journal of Psychiatry, 161(12), 2312-2314 https://doi.org/10.1176/appi.ajp.161.12.2312

Vilain, J., Galliot, A.-M., Durand-Roger, J., Leboyer, M., Llorca, P.-M., Schürhoff, F., \& Szöke, A. (2013). Les facteurs de risque environnementaux de la schizophrénie. L'Encéphale, 39(1), 19-28. https://doi.org/10.1016/j.encep.2011.12.007

Weich, S., Holt, G., Twigg, L., Jones, K., \& 
Lewis, G. (2003). Geographic variation in the prevalence of common mental disorders in Britain: A multilevel investigation. American Journal of Epidemiology, 1578), 730-737.

Wu, Y.-T., Prina, A. M., Jones, A., Matthews, F. E., \& Brayne, C. (2017). The Built Environment and Cognitive Disorders: Results From the Cognitive Function and Ageing Study II. American Journal of Preventive Medicine, 53(1), 25-32. https://doi.org/10.1016/j.amepre.2016.11.020

Zammit, S., Lewis, G., Rasbash, J., Dalman, C., Gustafsson, J.-E., \& Allebeck, P. (2010). Individuals, Schools, and Neighborhood: A Multilevel Longitudinal Study of Variation in Incidence of Psychotic Disorders. Archives of General Psychiatry, 679), 914. https://doi.org/10.1001/archgenpsychiatry.201 0.101 